Supporting Information

\title{
Well-defined, Nanostructured, Amorphous Metal Phosphate as Electrochemical Pseudocapacitor Materials with High Capacitance
}

Yaoning Xi, Bohua Dong*, Yanan Dong, Nan Mao, Lei Ding, Liang Shi, Rongjie

Gao, Wei Liu, Ge Su, Lixin Cao*

Institute of Materials Science and Engineering, Ocean University of China, 238

Songling Road, Qingdao, 266100 P. R. China 


\section{Supporting Figure}
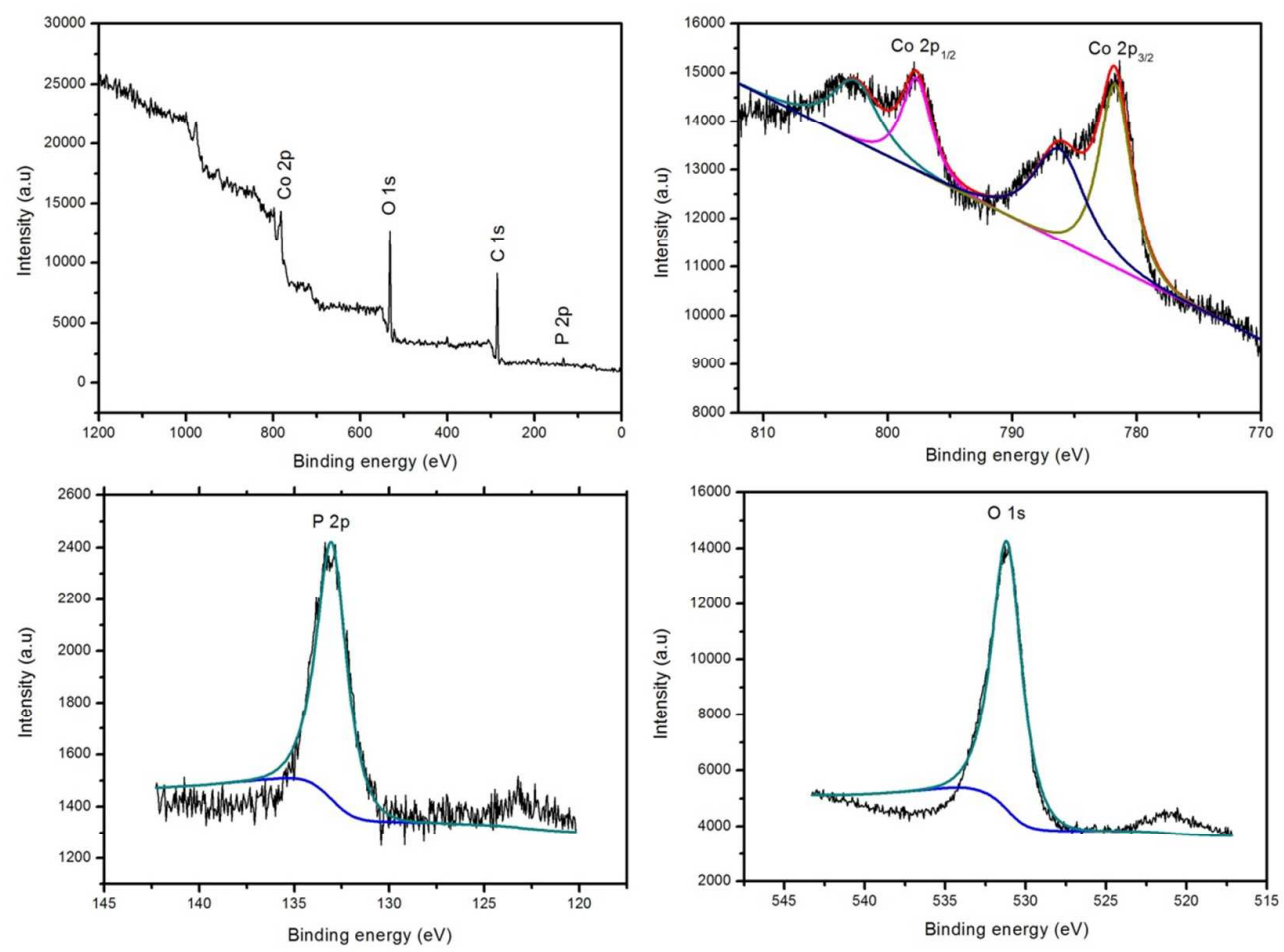

Figure S1. XPS spectrum of $\mathrm{Co}_{3}\left(\mathrm{PO}_{4}\right)_{2}$ nanosheet showing the composition of both Co, P and O. High-resolution XPS spectra of Co 2p, P 2p and O 1s.

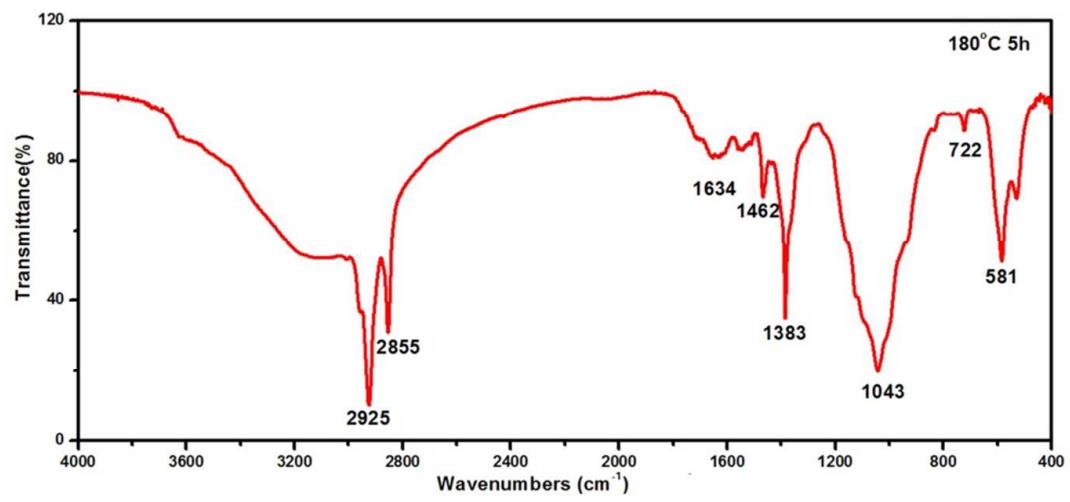

Figure S2. FTIR spectra of $\mathrm{Co}_{3}\left(\mathrm{PO}_{4}\right)_{2}$ nanosheet

The presence of oleylamine and $\mathrm{PO}_{4}{ }^{3-}$ in the amorphous $\mathrm{Co}_{3}\left(\mathrm{PO}_{4}\right)_{2}$ nanosheet was determined by FT-IR spectra. As shown in Figure S2, the triply degenerated asymmetric stretching and bending vibrations of $\mathrm{PO}_{4}{ }^{3-}$ were at $1050 \mathrm{~cm}-1$ and 570 
$\mathrm{cm}-1$. The two bands in the range of $3200-3300 \mathrm{~cm}^{-1}$ correspond to the $\mathrm{N}-\mathrm{H}$ stretching vibrations of the oleylamine. The bands located at about $2924 \mathrm{~cm}^{-1}$ and $2853 \mathrm{~cm}^{-1}$ correspond to the asymmetrical and symmetrical stretching vibration of $-\mathrm{CH}_{2}$, respectively. Intensive band at about $1620 \mathrm{~cm}^{-1}$ is attributed to the stretching vibration of $-\mathrm{C}=\mathrm{C}-$. Additionally, the bands located at about $1467 \mathrm{~cm}^{-1}$ and $1378 \mathrm{~cm}^{-1}$ correspond to the bending vibration of $-\mathrm{CH}_{3}$, and the band at $720 \mathrm{~cm}^{-1}$ corresponds to the bending vibration of $-\left(\mathrm{CH}_{2}\right)_{\mathrm{n}^{-}}$. All these bands analysis above can be ascribed to the vibration of oleylamine.

\begin{tabular}{|l|c|c|c|}
\hline \multicolumn{4}{|c|}{$180^{\circ} \mathrm{C}$ 5 hours ppm } \\
\hline Co_2286 & 10.25 & P_1774 & 7.877 \\
\hline Co_2378 & 9.524 & P_1782 & 7.729 \\
\hline Co_2388 & 11.03 & P_2136 & 4.869 \\
\hline Average & 6.15 & Average & 3.816 \\
\hline \multicolumn{4}{|c|}{ Co:P=1.6 } \\
\hline
\end{tabular}

Table S1. ICP results of $\mathrm{Co}_{3}\left(\mathrm{PO}_{4}\right)_{2}$ nanosheet

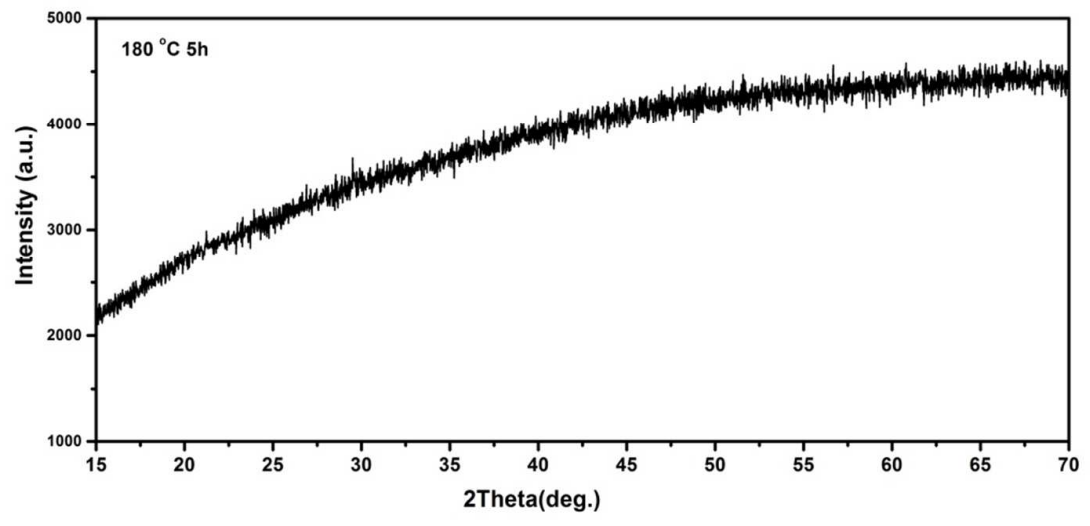

Figure S3. XRD pattern of $\mathrm{Co}_{3}\left(\mathrm{PO}_{4}\right)_{2}$ nanosheet. 

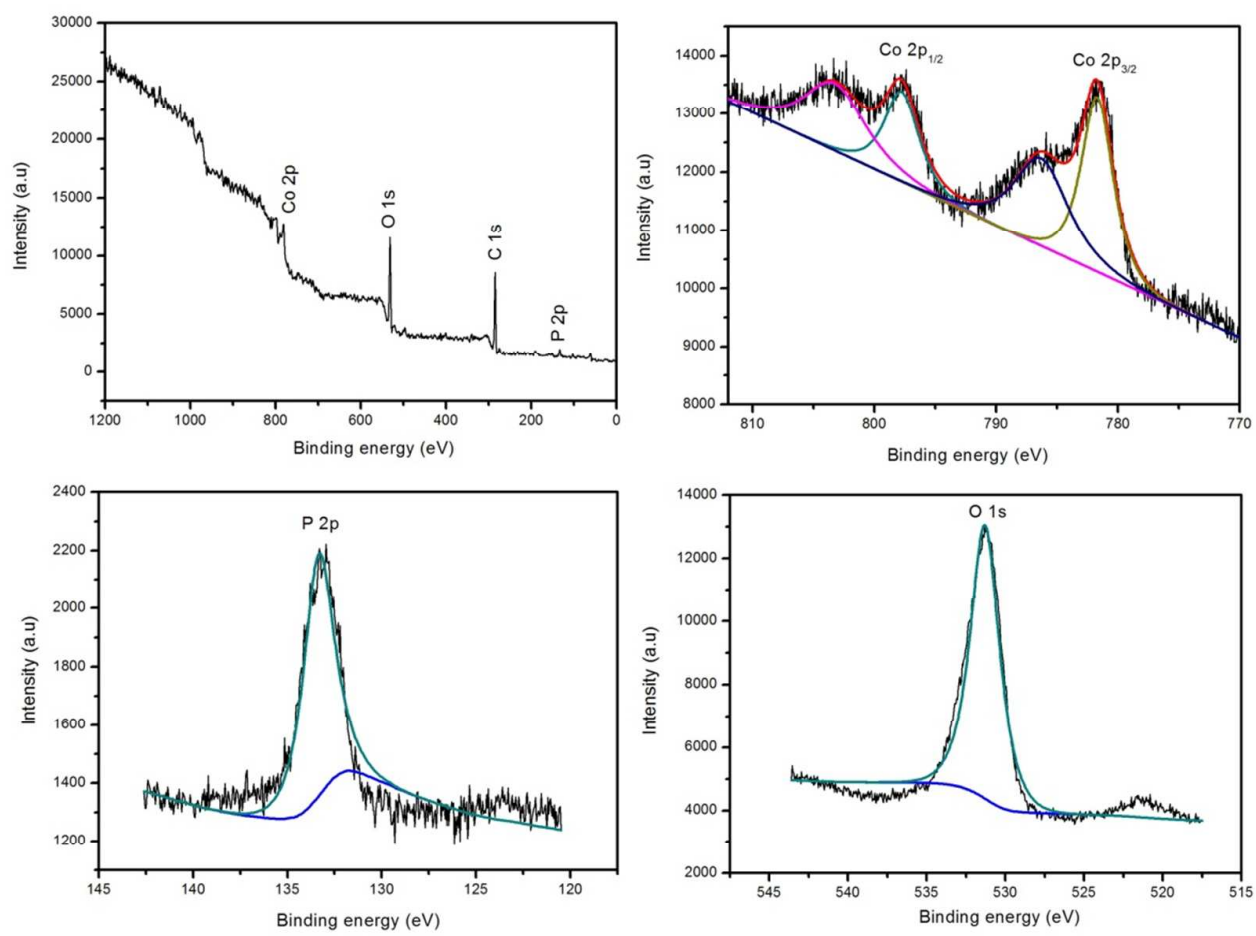

Figure S4 XPS spectrum of $\mathrm{Co}_{3}\left(\mathrm{PO}_{4}\right)_{2}$ nanowire showing the composition of both Co, $\mathrm{P}$ and O. High-resolution XPS spectra of Co 2p, P $2 p$ and O 1s.

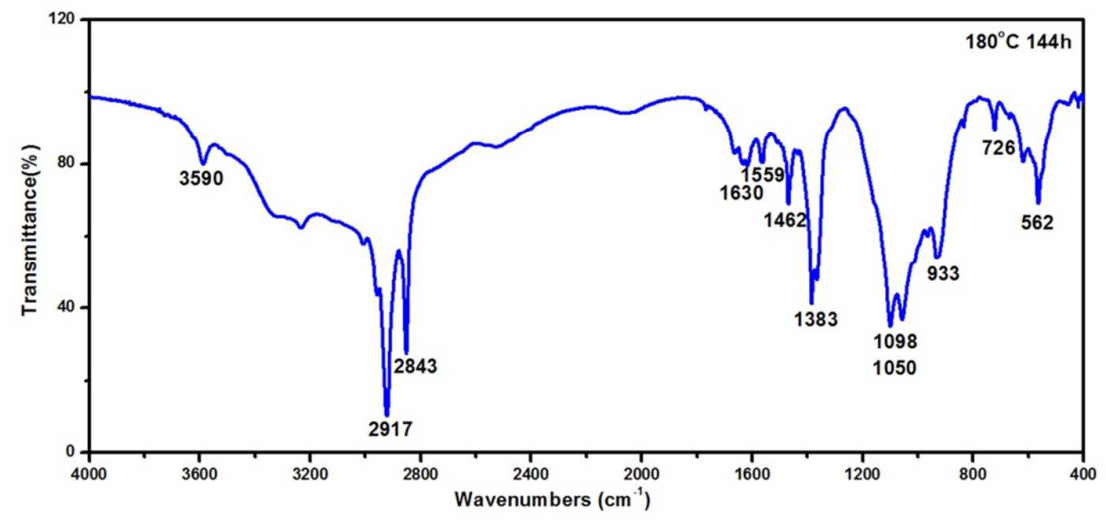

Figure S5. FTIR spectra of $\mathrm{Co}_{3}\left(\mathrm{PO}_{4}\right)_{2}$ nanowire

The presence of oleylamine and $\mathrm{PO}_{4}{ }^{3-}$ in the amorphous $\mathrm{Co}_{3}\left(\mathrm{PO}_{4}\right)_{2}$ nanosheet was determined by FT-IR spectra. As shown in Figure S2, the triply degenerated asymmetric stretching and bending vibrations of $\mathrm{PO}_{4}{ }^{3-}$ were at $1050 \mathrm{~cm}-1$ and 570 
$\mathrm{cm}-1$. The two bands in the range of $3200-3300 \mathrm{~cm}^{-1}$ correspond to the $\mathrm{N}-\mathrm{H}$ stretching vibrations of the oleylamine. The bands located at about $2924 \mathrm{~cm}^{-1}$ and $2853 \mathrm{~cm}^{-1}$ correspond to the asymmetrical and symmetrical stretching vibration of $-\mathrm{CH}_{2}$, respectively. Intensive band at about $1620 \mathrm{~cm}^{-1}$ is attributed to the stretching vibration of $-\mathrm{C}=\mathrm{C}-$. Additionally, the bands located at about $1467 \mathrm{~cm}^{-1}$ and $1378 \mathrm{~cm}^{-1}$ correspond to the bending vibration of $-\mathrm{CH}_{3}$, and the band at $720 \mathrm{~cm}^{-1}$ corresponds to the bending vibration of $-\left(\mathrm{CH}_{2}\right)_{\mathrm{n}^{-}}$. All these bands analysis above can be ascribed to the vibration of oleylamine.

\begin{tabular}{|l|c|c|c|}
\hline \multicolumn{4}{|c|}{$180^{\circ} \mathrm{C} 144$ hours ppm } \\
\hline Co_2286 & 6.147 & P_1774 & 4.172 \\
\hline Co_2378 & 5.691 & P_1782 & 4.323 \\
\hline Co_2388 & 6.602 & P_2136 & 2.953 \\
\hline Average & 10.268 & Average & 6.825 \\
\hline \multicolumn{4}{|c|}{ Co:P=1.5 } \\
\hline
\end{tabular}

Table S1. ICP results of $\mathrm{Co}_{3}\left(\mathrm{PO}_{4}\right)_{2}$ nanowire

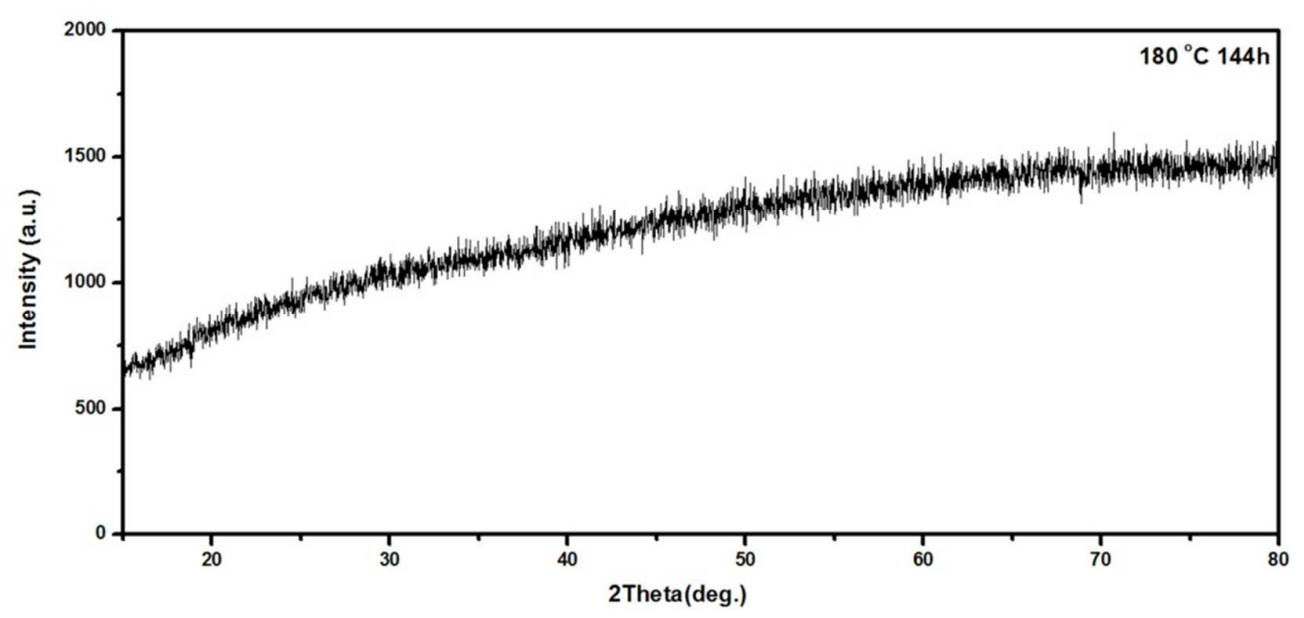

Figure S6. XRD pattern of $\mathrm{Co}_{3}\left(\mathrm{PO}_{4}\right)_{2}$ nanowire 

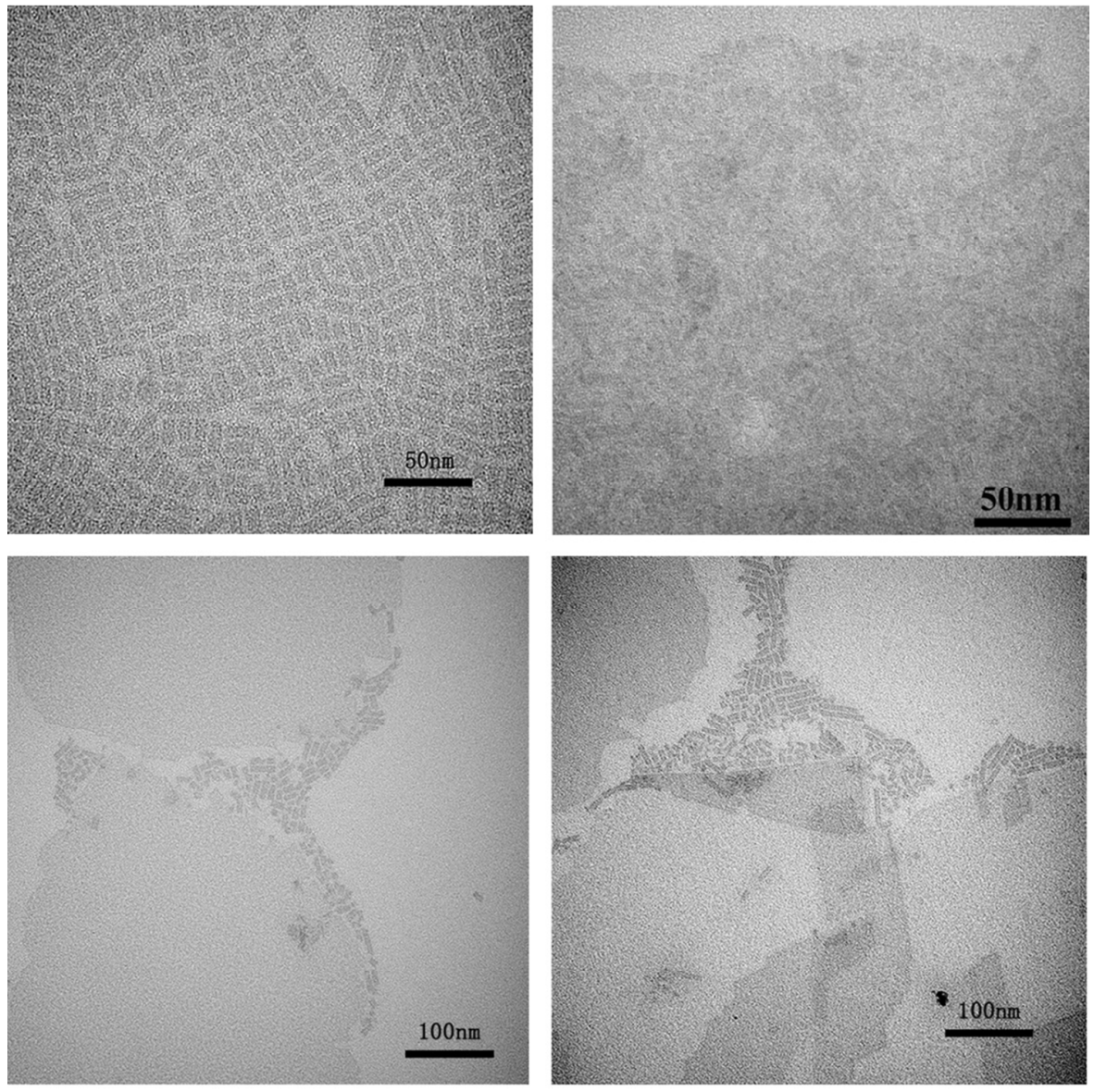

Figure S7. Detail TEM observation of the nanosheet generated by random attachment of nanoplate building blocks. We concluded that the initial-formed nanoplates were gradually consumed to be randomly attached and coalesce into the nanosheet as elongating the reaction time.
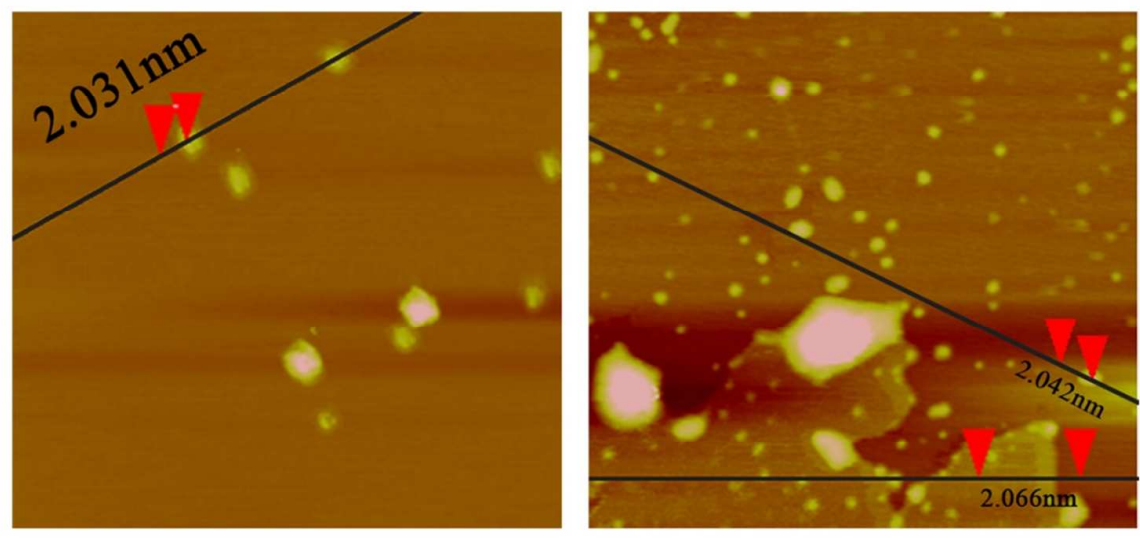
Figure S8. AFM images of nanoplate (left) and nanosheet (right). AFM analysis confirms that the thickness of nanoplate and nanosheet is consistent with each other, further supporting that the nansheet is assembly of nanoplates.
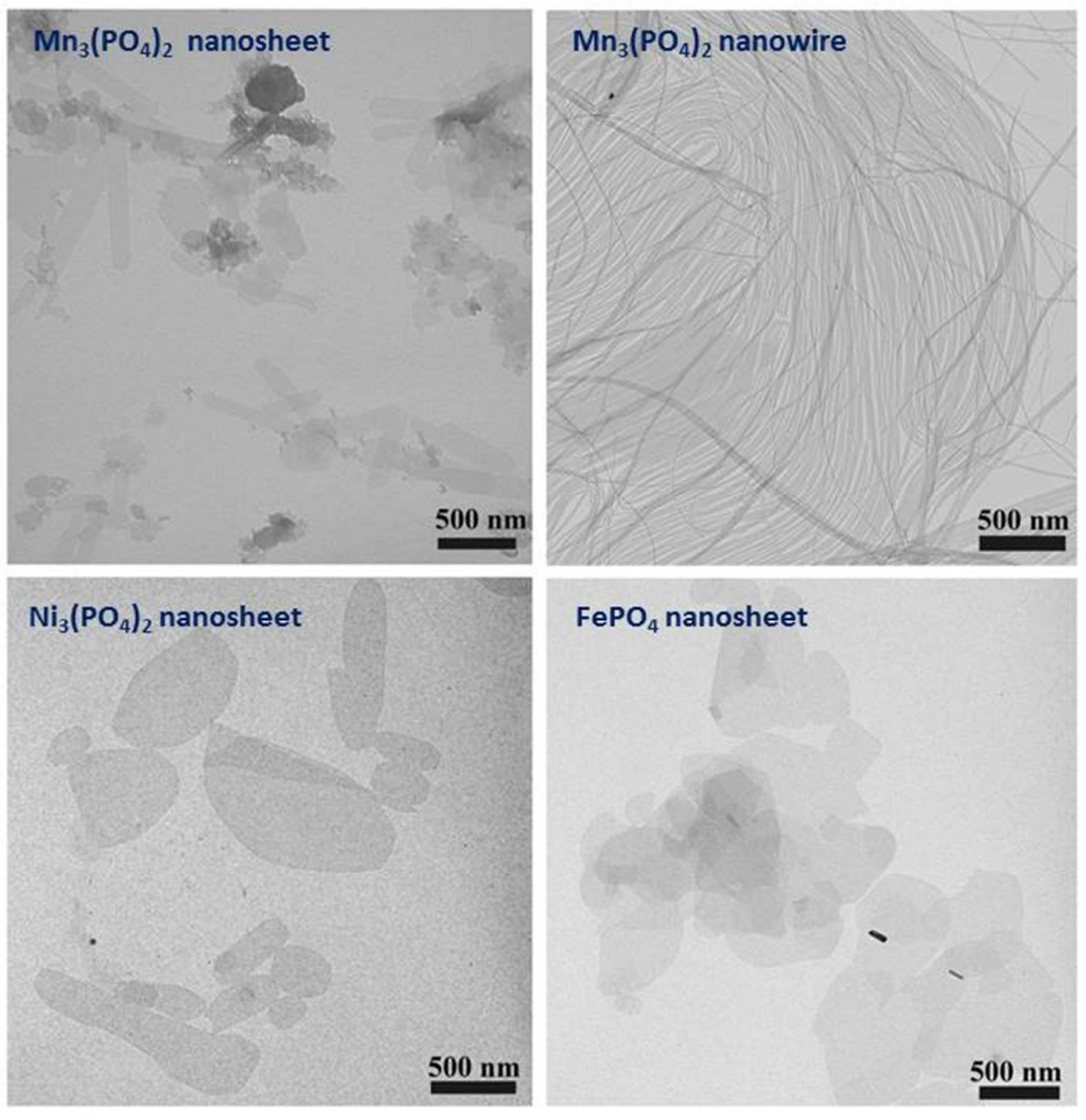

Figure S9. TEM images of metal phosphate amorphous ultrathin nanostructure 

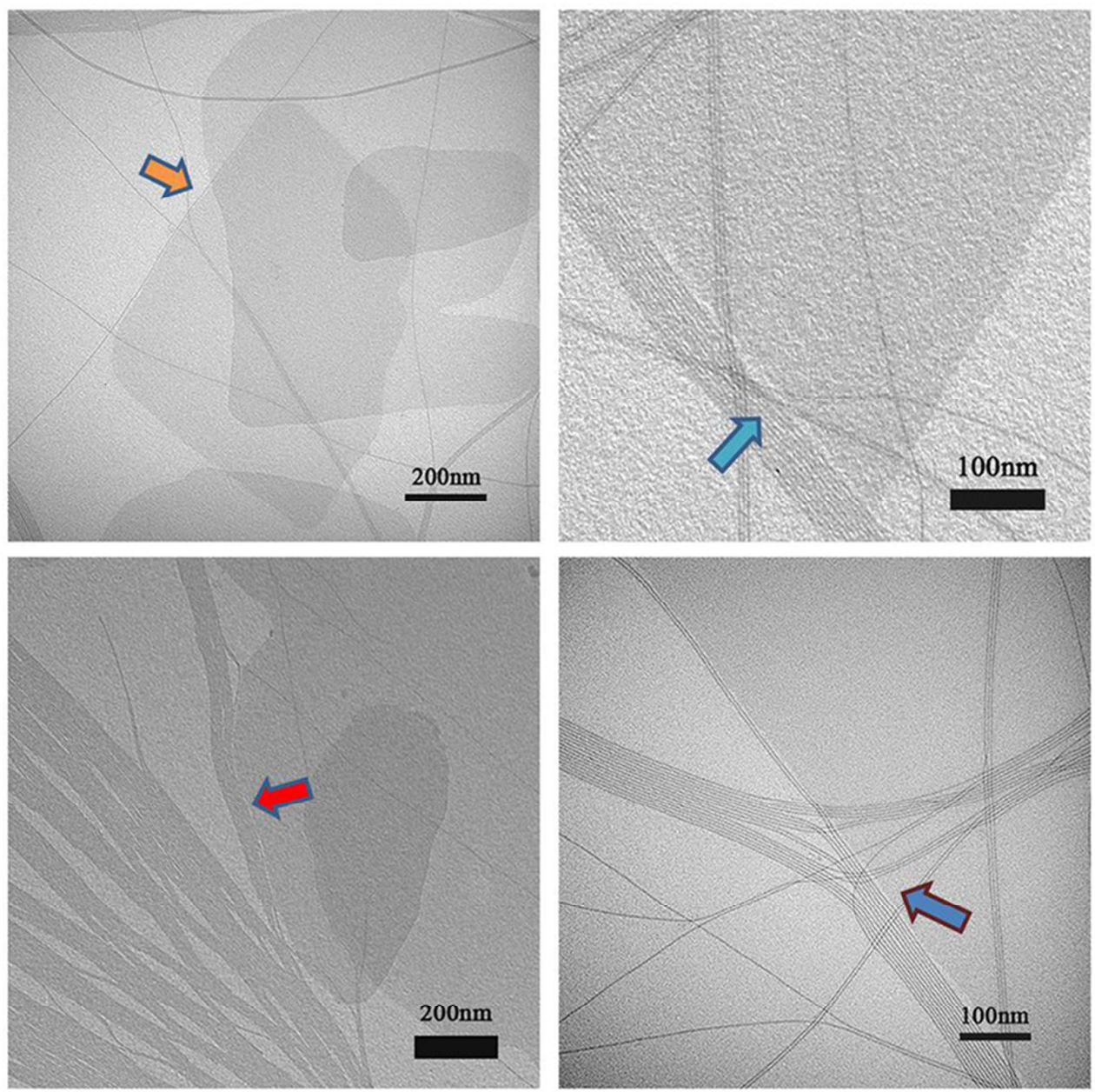

Figure S10. TEM images of nanowire split from nanosheet. 


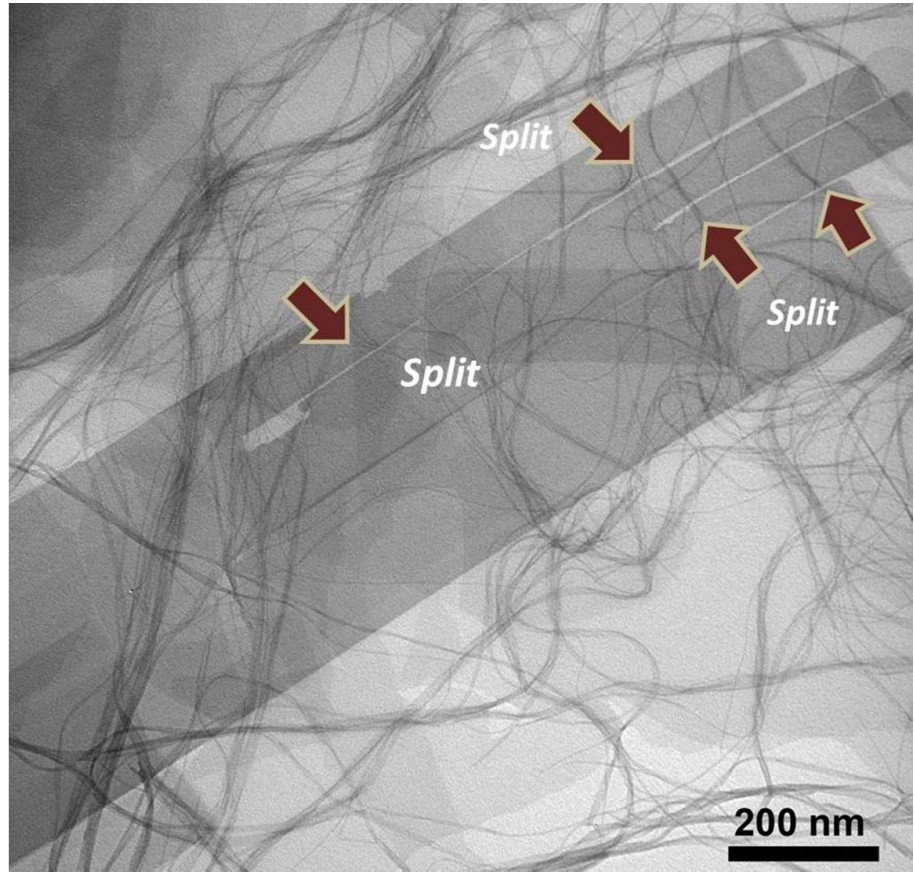

Figure S11. TEM images of nanosheet with obvious crack which could be facilitated by $\mathrm{OM}$, resulted in the split of the ultrathin nanowire from the nanosheet 


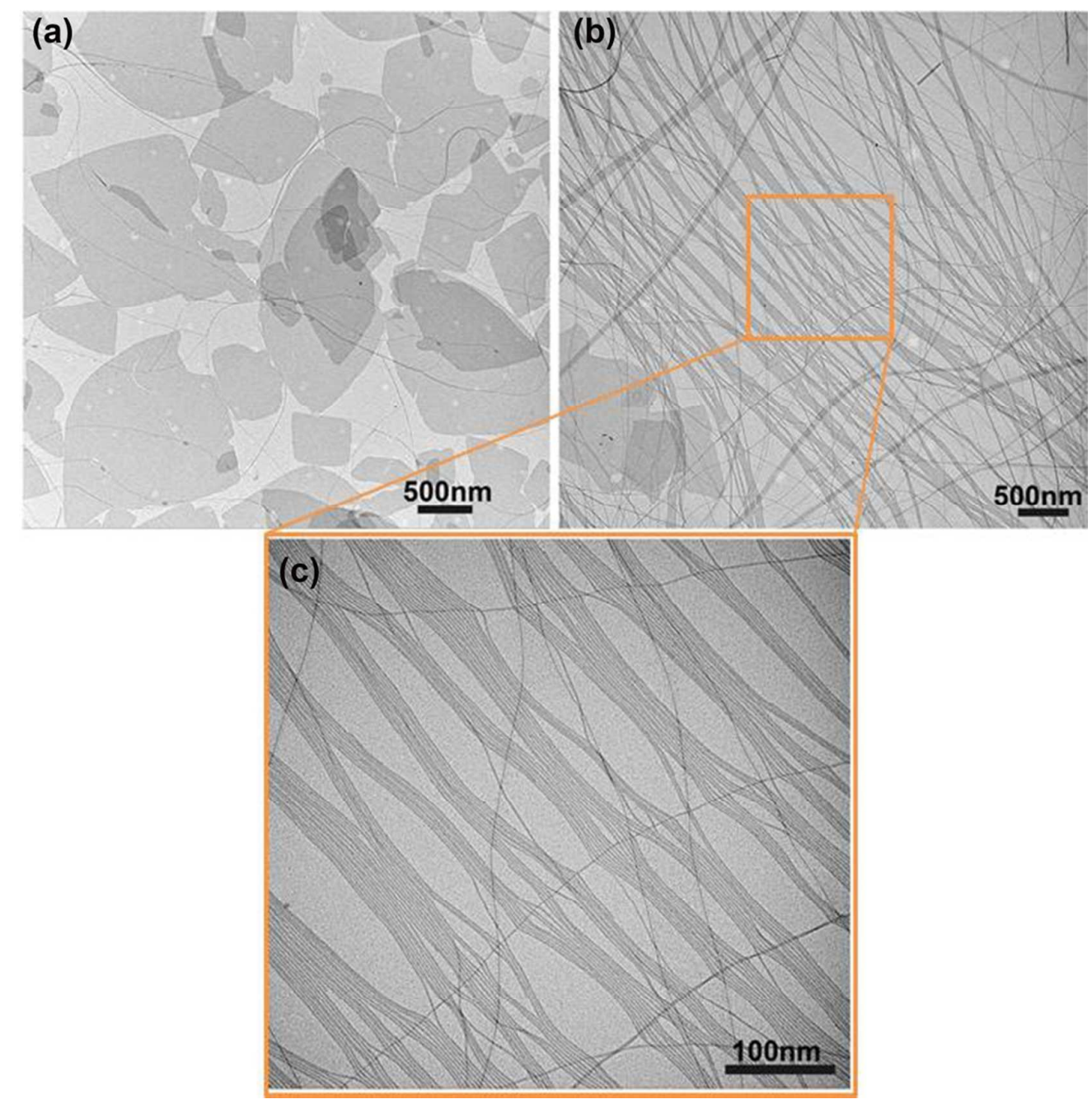

Figure S12. TEM images of $\mathrm{Co}_{3}\left(\mathrm{PO}_{4}\right)_{2}$ nanosheet aging with no $\mathrm{OM}$ and with $\mathrm{OM}$ in ODE. The nanosheet are first synthesized and purified to remove any unreacted monomers and excess $\mathrm{OM}$, and then the purified nanosheet are redispersed into octadecene and for further aging without OM. During the aging process, in the absence of OM, fewer nanowires are observed (a). However, by contrast, if the OM are added, the final majority of products are nanowire $(b, c)$. 


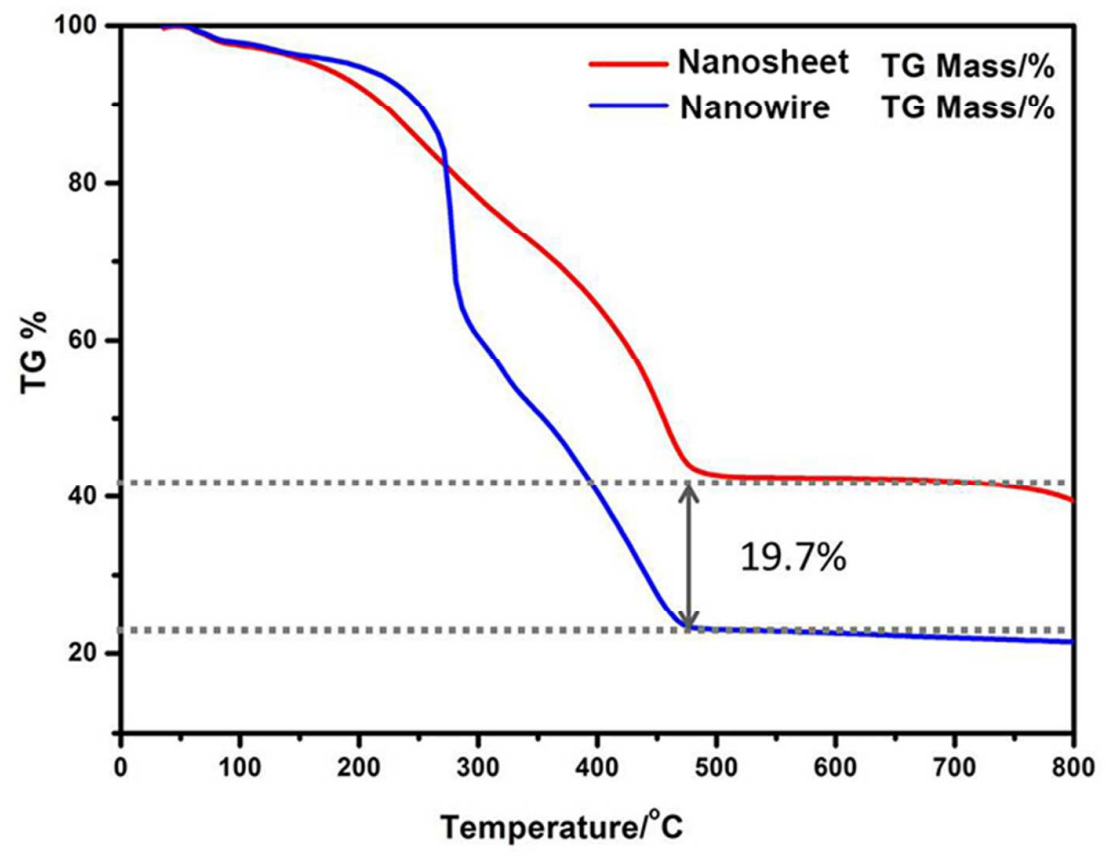

Figure S13. TG analysis of $\mathrm{Co}_{3}\left(\mathrm{PO}_{4}\right)_{2}$ nanowire and nanowsheet 

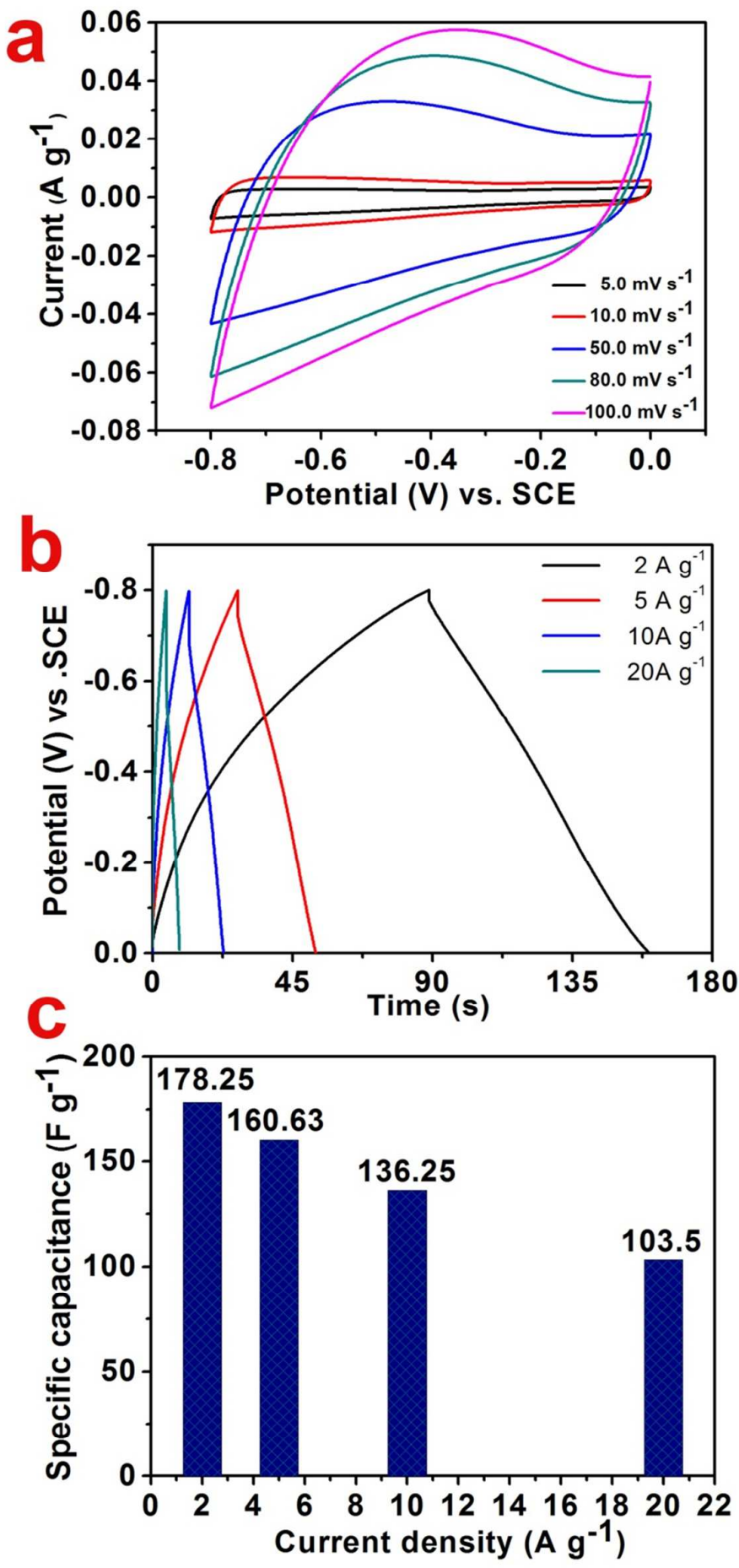

Figure S14. Electrochemical Characterization of active carbon electrode. (a) CV curves, (b) Charge-discharge curves and (c) Specific capacitance of the active carbon 